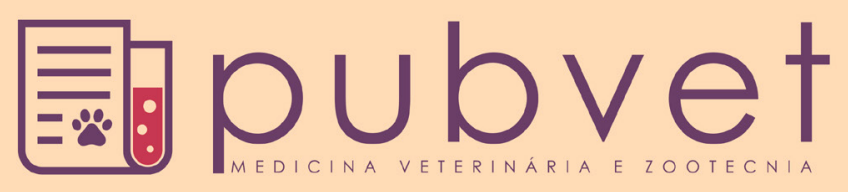

HTTP://DX.DOI.ORG/10.22256/PUBVET.V11N5.527-531

\title{
Comparação do peso corporal obtido através de pesagem em balança digital ou fita torácica de pesagem em Bovinos Nelore
}

\author{
André Hiroshi Quadros Watanabe ${ }^{1}$, Heloise Manchini², Wilmar Sachetin Marçal ${ }^{3 *}$ \\ ${ }^{I}$ Graduado em Medicina Veterinária, Universidade Estadual de Londrina, Londrina - PR. \\ ${ }^{2}$ Graduanda em Medicina Veterinária, Universidade Estadual do Norte do Paraná, Bandeirantes - PR. \\ ${ }^{3}$ Docente, Departamento de Clínicas Veterinárias, Universidade Estadual de Londrina, Caixa Postal 6001, CEP: \\ 86051-970, Londrina - PR. *Autor para correspondência: wilmar@uel.br
}

\begin{abstract}
RESUMO. A determinação do peso corporal dos animais é importante para avaliar o crescimento e o estado nutricional, administrar adequadamente medicamentos e parasiticidas, ajustar o arraçoamento e estabelecer o valor do animal para abate. No entanto, muitas são as propriedades que não dispõem de balanças para pesagem dos animais. Sendo assim, existem outras formas para avaliar o peso corporal sem o uso de balanças, realizado através de suas medidas corporais. As mais mencionadas na literatura são, o comprimento corporal, a altura da cernelha e da garupa e o comprimento da garupa. No presente estudo, investigou-se outro método empregado para avaliação do peso corporal sem a necessidade do uso de balança, a fita torácica para pesagem de bovinos, considerada um método indireto, de baixo custo e menos estressante. O objetivo deste trabalho foi verificar se haveriam diferenças entre os pesos obtidos nesses dois métodos, a fim de avaliar a eficácia do emprego da fita torácica de pesagem. Neste trabalho, avaliaram-se os pesos corporais de 46 machos Nelores de excelência zootécnica, com idade entre 15 a 17 meses, provindos da fazenda Cachoeira 2C, localizada no município de Sertanópolis - PR, obtidos através do emprego da fita torácica de pesagem para bovinos e pesagem em balança digital. Executando-se um Teste " $t$ " de Student, com um nível de significância de $5 \%$ a fim de comparar uma possível variação entre duas médias, sendo a primeira referente ao peso corporal obtido em balança digital $(\mathrm{P} 1)$, e a segunda referente à medição feita com a fita torácica de pesagem para bovinos $(\mathrm{P} 2)$, notou-se que houve variações na média $(\mathrm{P} 1=411,5$ / $\mathrm{P} 2=394,7)$, variância $(\mathrm{P} 1=1619,41111 / \mathrm{P} 2=2367,14058)$ e no desvio padrão $(\mathrm{P} 1=$ 40,24 / P2 = 48,65) e que, além disso, o valor de P foi maior que $0,05(0,074771791)$, ou seja, não houve diferenças entre os métodos comparados. Assim é possível afirmar que o uso da fita torácica para avaliação do peso corporal de bovinos é um método alternativo eficiente e de boa credibilidade, sendo uma ferramenta que pode ser empregada pelos produtores rurais com segurança.
\end{abstract}

Palavras-chave: Bovinos, perímetro torácico, peso corporal, medidas corporais, método indireto.

\section{Comparison of body weight obtained through weight in digital scales or thoracic weight tape in Nellore males}

ABSTRACT. The determination of the body weight of the animals is important to evaluate
the growth and the nutritional status, administer medications and parasiticides properly,
adjust the feed and establish the value of the animal for slaughter. However, since there are
many properties that do not have scales for weighing the animals. Therefore, there are other
ways to evaluate body weight without the use of scales, performed through their body
measurements. The most mentioned in the literature are body length, height of the withers
and croup, and length of the croup. In the present study, another method used to evaluate 
body weight without the use of a scales, the thoracic tape for bovine weighing, was considered an indirect, low cost and less stressful method. So the objective was to verify if there were differences between the weights obtained in these two methods, in order to evaluate the effectiveness of the use of the thoracic weighing tape. In this study, we evaluated the body weights of 46 Nellore males of zootechnical excellence, aged 15 to 17 months, coming from the farm Cachoeira 2C, located in the city of Sertanópolis - PR, obtained through the use of thoracic weighing tape for cattle And digital weighing. A Student's t-test was performed, with a significance level of 5\% (0.05) in order to compare a possible variation between two means, the first one referring to the body weight obtained in digital scale $(\mathrm{P} 1)(\mathrm{P} 1=411.5 / \mathrm{P} 2=394.7)$, variance $(\mathrm{P} 1=1619.41111 / \mathrm{P} 2)$ was observed, and the second one referring to the measurement made with the thoracic weighing tape for cattle $(\mathrm{P} 2)=2367.14058)$ and in the standard deviation $(\mathrm{P} 1=40.24 / \mathrm{P} 2$ $=48.65)$ and that in addition, the $\mathrm{P}$ value was greater than 0.05 (0.074771791), that is, there were no significant differences Between the methods compared. Thus, it is possible to affirm that the use of thoracic tape to evaluate the body weight of cattle is an efficient and credible alternative method, being a tool that can be used by farmers safely.

Keywords: Body measurements, Body weight, cattle, chest circumference, indirect method

\section{Comparación del peso corporal en machos Nelore obtenido con báscula digital o cinta torácica de pesaje}

RESUMEN. La determinación del peso corporal de los animales es importante para evaluar el crecimiento y estado nutricional, administración apropiada de medicamentos y parasiticidas, ajustar la alimentación y establecer el valor del animal para el sacrificio. Sin embargo, son muchas las granjas que no disponen de básculas para pesar los animales. Con todo, hay otras maneras de evaluar el peso corporal sin el uso de la báscula, realizado mediciones corporales. Las comúnmente mencionadas en la literatura son, la longitud corporal, la altura de la cruz y la grupa y la longitud de la grupa. En el presente estudio, se investigó otro método empleado para evaluar el peso corporal sin el uso de báscula, la cinta torácica para pesar bovinos, considerado un método indirecto, de bajo costo y menos estresante. El objetivo de este estudio fue verificar si existían diferencias entre los pesos obtenidos entre estos dos métodos, con el fin de evaluar la eficacia de utilización de la cinta de pesaje torácica. Se evaluó el peso corporal de 46 machos Nelore de excelencia zootécnica, con edad entre 15 a 17 meses, procedentes de la hacienda cascada 2C, localizada en la ciudad de Sertanópolis - PR, obtenidos usando la cinta torácica de pesaje para ganado y báscula digital. Se realizó la prueba t de Student, con un nivel de $5 \%$ de significancia para comparar la posible variación entre las dos medias, el primero haciendo referencia al peso corporal obtenido en báscula digital (P1), y la segunda medida haciendo referencia a los datos obtenidos con la cinta torácica de pesaje para bovinos $(\mathrm{P} 2)$, se notó que hubo variaciones en el promedio $(\mathrm{P} 1=411,5 / \mathrm{P} 2=394,7)$, varianza $(\mathrm{P} 1=1619,41111$ $/ \mathrm{P} 2=2367,14058)$ y en el desvió estándar $(\mathrm{P} 1=40,24 / \mathrm{P} 2=48,65)$ y que, además de eso el valor de $\mathrm{P}$ fue mayor que $0,05(0,074771791)$, es decir, no hubo diferencias entre los métodos comparados. Así, es posible afirmar que el uso de la cinta para evaluar el peso corporal torácico de ganado es un método alternativo eficaz y creíble, siendo una herramienta que puede ser usada de manera segura por los agricultores.

Palabras clave: Ganado, perímetro torácico, el peso corporal, medidas del cuerpo, método indirecto

\section{Introdução}

Estima-se que o Brasil possua um efetivo bovino aproximado de 212,3 milhões de animais, sendo o segundo colocado no ranking mundial, atrás apenas da Índia, mostrando assim a grande representatividade do país no mercado mundial de produtos de origem bovina (ANUALPEC, 2016, FAPRI, 2016). Devido a essa representatividade 
faz-se necessário à uniformização de metodologias para avaliação do animal vivo, carcaças e carne produzida.

A determinação do peso corporal dos animais é importante para avaliar o crescimento e o estado nutricional, administrar adequadamente medicamentos e parasiticidas, ajustar o arraçoamento e estabelecer o valor do animal para abate (Reis et al., 2008, Pereira et al., 2010). Entretanto, muitas propriedades não possuem balanças disponíveis para a pesagem dos animais e, muitas das vezes a opção é pela adoção de um método rápido e indireto que possa estimar o peso corporal. Além disso, há outros fatores que limitam o uso das balanças como exemplo, o estresse dos animais. Sendo assim, uma forma de avaliação do peso corporal dos animais sem o uso de balanças pelas suas medidas corporais. As mais mencionadas na literatura para predizer o peso são o perímetro torácico, o comprimento corporal, a altura da cernelha e da garupa e o comprimento da garupa. Embora existam discrepâncias sobre qual medida individual deve ser utilizada para predizer o peso, a acurácia da predição tem sido geralmente alta especialmente quando mais de uma medida for considerada. Estas estimativas podem ser influenciadas pela raça, idade, condição corporal e estado fisiológico do animal (Khalil and Vaccaro, 2002, Heinrichs et al., 1992).

Um método indireto de pesagem de animais pode ser realizado através da utilização da fita torácica de pesagem, sendo uma ferramenta mais barata e menos estressante ao animal, sem contar que é mais prática, pois pode ser utilizado em qualquer local. O método da fita torácica consiste em uma fita de medição, colocada em torno da circunferência do tórax do animal, sendo que as equações de estimativa do peso pelo perímetro torácico correspondem a um peso vivo estimado a partir de medidas de 5.723 novilhas em fazendas comerciais na Pensilvânia - EUA ( $\underline{2010)}$.

Por se tratar de uma estimativa, diversos estudos foram realizados com o intuito de avaliar a eficácia e credibilidade da fita torácica de pesagem. Setim et al. (2010) comparando os pesos dos animais em balança mecânica com os obtidos com a fita torácica, verificaram diferenças que variaram de 5,0 a $12 \mathrm{~kg}$ superiores para a medida com a fita torácica, bem como de 1,0 a $8,0 \mathrm{~kg}$ inferiores para a fita. Apesar de demonstrarem pequena variação no peso dos animais, os autores concluíram que a fita torácica é uma excelente ferramenta para obtenção do peso dos animais, principalmente em pequenas propriedades, visto que o custo de uma balança mecânica ou digital se torna inviável para pequenas propriedades.

Em se tratando do peso corporal para comercialização do animal, não haverá problemas uma vez que os animais serão devidamente pesados em balança mecânica ou digital nos abatedouros. Contudo, ao se realizar cálculos referentes a nutrientes e, principalmente, para administração de medicamentos, as diferenças no peso corporal podem levar a quadros de sub ou superdosagem e, neste caso colocar em risco a vida do animal, principalmente em se tratando de medicamentos que apresentam baixo índice terapêutico e, portanto, alto índice de toxicidade (Abreu et al., 2015).

O objetivo deste trabalho é verificar se existem diferenças significativas entre os pesos corporais obtidos a partir da aferição com fita torácica de pesagem e com balança digital, avaliando-se os respectivos pesos vivos de machos da raça Nelore, buscando determinar se a fita torácica de pesagem para bovinos é uma ferramenta que pode ser empregada com segurança pelos produtores rurais.

\section{Material e Métodos}

A pesquisa foi realizada na fazenda Cachoeira 2C, com gado de excelência zootécnica, localizada no município de Sertanópolis (Área de 505,53 $\mathrm{km}^{2}$, Latitude: -23.0597, Longitude: $-51.033223^{\circ}$ 3' $35^{\prime \prime}$ Sul, $51^{\circ} 1^{\prime} 60^{\prime \prime}$ Oeste), estado do Paraná. Ao todo, foram avaliados os pesos corporais de 46 (quarenta e seis), machos da raça Nelore, com idade média entre 15 e 17 meses, criados sob manejo semiextensivo.

Os animais foram contidos adequadamente em tronco de contenção específico para a espécie bovina, não causando estresse demasiado a estes animais. Avaliou-se o peso vivo dos animais através de pesagem em balança eletrônica digital, da marca Beckhauser ${ }^{\circledR}$ (1), e com utilização de fita torácica de pesagem para bovinos, da marca Zolla ${ }^{\circledR}$ (2), sendo realizada caudalmente à escápula passando pelo esterno e pelos processos espinhais das vértebras torácicas. Vale ressaltar que antes de cada aferição a balança digital era devidamente zerada para que assim não houvesse intercorrências durante a pesagem, interferindo no valor final.

Os resultados foram avaliados utilizando-se o Teste " $t$ " de Student, para analisar as possíveis variações entre o uso de balança digital e fita 
torácica de pesagem. Considerando-se um nível de significância de $5 \%(0,05)$. Quando o valor do $\mathrm{P}$ for menor do que 0,05 rejeita-se a hipótese nula, ou seja, afirma-se que há diferença entre os pesos obtidos com o emprego da balança digital e da fita torácica de pesagem (Ferreira, 2011).

\section{Resultados e Discussão}

$\mathrm{Na}$ Tabela 1 apresentam-se os pesos corporais de cada um dos 46 animais avaliados neste trabalho. Nota-se que P1 (peso 1), refere-se ao peso corporal obtido em balança digital e $\mathrm{P} 2$ (peso 2), ao peso obtido através da fita torácica de pesagem para bovinos.

Tabela 1. Valores do peso corporal, em quilogramas, dos 46 machos Nelores avaliados, obtidos pela utilização de balança digital (P1) e fita torácica de pesagem para bovinos (P2), Sertanópolis - PR

\begin{tabular}{|c|c|c|}
\hline Animal & $\mathrm{P} 1$ & $\mathrm{P} 2$ \\
\hline 1 & 453 & 479 \\
\hline 2 & 445 & 461 \\
\hline 3 & 409 & 470 \\
\hline 4 & 464 & 448 \\
\hline 5 & 457 & 440 \\
\hline 6 & 524 & 531 \\
\hline 7 & 371 & 413 \\
\hline 8 & 439 & 380 \\
\hline 9 & 414 & 407 \\
\hline 10 & 407 & 440 \\
\hline 11 & 456 & 440 \\
\hline 12 & 458 & 387 \\
\hline 13 & 414 & 375 \\
\hline 14 & 453 & 420 \\
\hline 15 & 409 & 361 \\
\hline 16 & 414 & 361 \\
\hline 17 & 411 & 333 \\
\hline 18 & 422 & 407 \\
\hline 19 & 429 & 448 \\
\hline 20 & 374 & 413 \\
\hline 21 & 488 & 484 \\
\hline 22 & 444 & 407 \\
\hline 23 & 451 & 427 \\
\hline 24 & 406 & 339 \\
\hline 25 & 395 & 387 \\
\hline 26 & 416 & 387 \\
\hline 27 & 400 & 326 \\
\hline 28 & 382 & 380 \\
\hline 29 & 495 & 470 \\
\hline 30 & 374 & 333 \\
\hline 31 & 432 & 394 \\
\hline 32 & 356 & 349 \\
\hline 33 & 379 & 375 \\
\hline
\end{tabular}

\section{Tabela1. Continuação}

\begin{tabular}{lll}
\hline Animal & P1 & P2 \\
\hline 34 & 360 & 339 \\
25 & 395 & 387 \\
34 & 360 & 339 \\
35 & 373 & 375 \\
36 & 371 & 394 \\
37 & 388 & 344 \\
38 & 369 & 387 \\
39 & 380 & 355 \\
40 & 392 & 361 \\
41 & 351 & 349 \\
42 & 363 & 326 \\
43 & 382 & 380 \\
44 & 404 & 387 \\
45 & 399 & 355 \\
46 & 356 & 333 \\
\hline
\end{tabular}

Fonte: O autor (2016).

A fim de comprovar os resultados obtidos, executou-se o Teste " $t$ " de Student, sendo indicado para comparar uma possível variação entre duas médias, sendo a primeira referente ao peso corporal em balança digital, e a segunda referente ao peso corporal aferido através da fita torácica de pesagem para bovinos.

$\mathrm{Na}$ Tabela 2 apresentam-se os valores de média, variância, desvio padrão e $P$ value analisados pelos pesos corporais dos 46 machos Nelores avaliados.

De acordo com os valores explicitados na Tabela 2 , nota-se que houve variações na média, variância e no desvio padrão. Além disso, o $\mathrm{P}$ vale 0,074771791, sendo maior que 0,05 (nível de significância de 5\%).

Tabela 2. Valores de média, variância, desvio padrão e $P$ value, dos 46 machos Nelores avaliados, obtidos através da utilização de balança digital (P1) e fita torácica de pesagem para bovinos (P2), Sertanópolis - PR

\begin{tabular}{lcc}
\hline & $\mathrm{P} 1$ & $\mathrm{P} 2$ \\
\hline Média* & 411,5 & 394,7 \\
Variância & 1619,41111 & 2367,14058 \\
Desvio Padrão* & 40,24 & 48,65 \\
\hline $\mathrm{P}(\mathrm{T}<=\mathrm{t})$ bi-caudal & \multicolumn{2}{c}{0,074771791} \\
\hline *Valores expressos em quilogramas. Fonte: O autor (2016).
\end{tabular}

Portanto é possível afirmar que não houve diferença entre os pesos obtidos nos dois métodos de aferição, balança digital e fita torácica. Tal resultado condiz com o exposto por Setim et al. (2010) em seu trabalho, afirmando que embora houvesse uma pequena variação no peso dos animais, os autores concluíram que a fita torácica 
é uma excelente ferramenta para obtenção do peso dos animais.

\section{Conclusão}

Com base nas análises dos dados estatísticos é possível concluir que a adoção da fita torácica de pesagem para bovinos mostra-se como um método indireto eficiente e de boa credibilidade, não havendo variação significativa entre os pesos dos animais obtidos com o emprego da fita torácica e balança digital, sendo uma ferramenta acessível a pequenos produtores rurais devido a seu baixo custo, além da praticidade em sua utilização, sendo menos estressante aos animais.

\section{Referências Bibliográficas}

Abreu, B. A., Magalhães, C. J., Duayer, E., Machado, S. H. M. \& Silva, D. A. 2015. Variação da medida torácica obtida com a fita métrica tradicional com fator de correção e com a fita de pesagem para bovinos. Acta Biomedica Brasiliensia, 6, 42-48.

ANUALPEC. 2016. Anuário da Pecuária Brasileira, 20th edn. Instituto FNP, São Paulo, SP, Brasil.

FAPRI. 2016. Food and Agricultural Policy Research Institute. In: Database, W. A. O. (ed.) Food and Agricultural Policy Research Institute. Iowa State University and University of Missouri-Columbia Ames, IA, USA.

Ferreira, D. F. 2011. SISVAR: A Computer Statistical Analysis System. Ciência e Agrotecnologia, 35, 1039-1042.

Heinrichs, A. J., Rogers, G. W. \& Cooper, J. B. 1992. Predicting body weight and wither height in Holstein heifers using body measurements. Journal of Dairy Science, 75, 3576-3581.
Khalil, R. \& Vaccaro, L. 2002. Body weights and measurements in dual purpose cows: their interrelation and association with genetic merit for three production traits. Zootecnia Tropical, 20, 11-30.

Pereira, E. S., Pimentel, P. G., Queiroz, A. C. \& Mizubuti, I. Y. 2010. Novilhas leiteiras. Graphiti Gráfica e Editora Ltda, Fortaleza, Ceará.

Reis, G. L., Albuquerque, F. H. M. A. R., Valente, B. D., Martins, G. A., Teodoro, R. L., Ferreira, M. B. D., Monteiro, J. B. N., Silva, M. A. \& Madalena, F. E. 2008. Predição do peso vivo a partir de medidas corporais em animais mestiços Holandês/Gir. Ciência Rural, 38, 778-783.

Setim, D.H.; Vanzan, M; Fernandes, M.V; Donicht, A.M.M. 2010. Comprovação da eficácia do uso da fita torácica de pesagem em bovinos leiteiros. Disponível em: < http://www.cafw.ufsm.br/mostraciencias/2011 /resumos/210.pdf $>$ Acesso em 22 de jun. 2016.

\section{Article History:}

Received 11 January 2017'

Accepted 23 January 2017

Available on line 27 March 2017

License information: This is an open-access article distributed under the terms of the Creative Commons Attribution License 4.0, which permits unrestricted use, distribution, and reproduction in any medium, provided the original work is properly cited. 